\title{
Reemplazo de la estabilización tartárica por frío en las bodegas: El uso de carboximetil celulosa, poliaspartato de potasio y resinas intercambiadoras de iones
}

\section{Replacement of wine cold stabilization in the wineries: The use of carboximethyl cellulose, potassium polyaspartate and ion exchange resins}

\author{
P. Martínez-Pérez, A.B. Bautista Ortín, V. Durand, y E. Gómez-Plaza \\ Departamento de Tecnología de Alimentos, Nutrición y Bromatología, Universidad de Murcia, Campus de Espinardo, \\ 30100 Murcia, España
}

\begin{abstract}
Resumen. El tratamiento de los vinos antes del embotellado para evitar la precipitación de las sales del ácido tartárico es un paso importante y común durante la producción del vino. La estabilización en frío es el tratamiento de estabilización más utilizado, y aunque ha demostrado ser efectiva, presenta algunas desventajas significativas. Estas son las razones por las cuales se están introduciendo otros productos y metodologías en las bodegas. Algunas de estas nuevas técnicas implican la reducción de la concentración de ácido tartárico y/o potasio en los vinos. Otras son técnicas aditivas y utilizan coloides protectores o inhibidores de la cristalización de las sales. En este estudio, se han tratado con carboximetilcelulosa, con poliaspartado de potasio y con resina de intercambio iónico vinos blancos, rosados y dos tipos diferentes de vinos tintos. Se estudiaron las características enológicas y cromáticas de los vinos y se evaluaron también sensorialmente. Los resultados indican que las características sensoriales y cromáticas se mantuvieron en los vinos (en comparación con un vino de control no tratado) con el uso de la carboximetilcelulosa y el poliaspartato, no detectándose diferencias en la mayoría de los casos en una prueba sensorial triangular en el caso de la carboximetilcelulosa y siendo los vinos tratados con poliaspartato de potasio evaluados positivamente.
\end{abstract}

\begin{abstract}
The treatment of wines before bottling to avoid the precipitation of tartaric acid salts is an important and common step during wine production. Cold stabilization is the most common stabilization treatment. Although it has been shown to be effective, it has some significant disadvantages. These are the reasons why other products and methodologies are being introduced in the wineries for the replacement of the cold stabilization process. Some of these new techniques involve the reduction of the concentration of tartaric acid and/or potassium in wines. Others are additive techniques and use protective colloids or inhibitors of the crystallization of salts. In this study, white, rosé wines and two different types of red wines have been treated with carboxymethylcellulose, potassium polyaspartate and an ion exchange resin. The oenological and chromatic characteristics of the wines were studied, and they were also evaluated sensorially. The results indicate that the sensory and chromatic characteristics were maintained in the wines (in comparison with an untreated control wine) with the use of carboxymethylcellulose and polyaspartate, with no differences being detected in most cases in a triangular sensory test.
\end{abstract}

\section{Introducción}

El bitartrato de potasio y el tartrato de calcio son sales del ácido tartárico presentes de forma natural en el vino, y que se vuelven cada vez más insolubles por la presencia de etanol y durante el posterior almacenamiento del producto embotellado a bajas temperaturas. Además, este problema se ve agravado en los últimos años porque un incremento en las temperaturas medias durante la maduración de las uvas provoca que éstas presenten menor acidez total en el momento de la vendimia y niveles mas altos de cationes, sobre todo potasio. Los cristales de sales del ácido tartárico no son un problema que ponga en peligro la salud del consumidor, pero pueden ser un problema para la aceptación por parte de éste y un problema legal en algunos países. Por lo tanto, el tratamiento de los vinos antes del embotellado para evitar la precipitación de estas sales es un paso importante y común durante la producción del vino.

La estabilización en frío es el tratamiento de estabilización más extendido y utilizado hoy día por las bodegas para alcanzar la estabilización tartárica del vino [1]. Consiste en su refrigeración a una temperatura cercana a la temperatura de congelación, introduciéndolo a continuación en un depósito isotérmico, y dejándolo durante un tiempo variable de 7-12 días para los blancos y rosados, y de algunas semanas para los tintos. Aunque la estabilización en frío ha demostrado ser efectiva, presenta algunas desventajas significativas, como el tiempo de 
tratamiento necesario, el alto costo económico de la técnica, la pérdida de materia colorante y compuestos responsables del aroma y sabor de los vinos, y los problemas ambientales por los residuos que se generan [2].

Estas son las razones por las cuales se están introduciendo otros productos y metodologías en las bodegas. Algunas técnicas se consideran "sustractivas" e implican fundamentalmente la reducción de la concentración de cationes potasio en los vinos. Otras son técnicas "aditivas" y utilizan coloides protectores o inhibidores de la cristalización que se pueden agregar al vino.

Entre los procesos sustractivos encontramos la utilización de resinas de intercambio catiónico (RIC) y la electrodiálisis. Esta última técnica necesita una inversión muy elevada ya que el equipamiento es extremadamente complejo y los costes de proceso y mantenimiento son elevados. En el caso de los sistemas de intercambio catiónico, el principio de funcionamiento se basa en el intercambio de cationes disueltos en un medio líquido por otros cationes (fundamentalmente de la misma carga) soportados sobre un lecho fijo. En el caso que nos ocupa, se trata de capturar cationes $\mathrm{K}+\mathrm{y}$ sustituirlos por protones $(\mathrm{H}+)$. De este modo, se evita la formación de bitartrato potásico, favoreciendo la presencia de ácido tartárico [2]. Una característica importante de la resina utilizada es que debe ser selectiva, eliminando el catión que se desea y respetando al máximo otros cationes presentes en el medio. Existen una serie de requisitos en su utilización como es que el tratamiento no debe hacer bajar el $\mathrm{pH}$ del vino a menos de 3.0 y la disminución no debe exceder 0.3 unidades de $\mathrm{pH}$ y la resina no debe transmitir al vino materias o características que normalmente no existen en el vino.

Entre los procesos aditivos encontramos la carboximetilcelulosa (CMC) y el poliaspartato de potasio (PA), cuyo uso ha sido recientemente aprobado por la OIV. Estos compuestos inhiben la cristalización del ácido tartárico.

La carboximetilcelulosa (CMC) o goma de celulosa es un polisacárido que se obtiene como celulosa modificada a partir de células vegetales. Los polímeros obtenidos tienen diferente solubilidad dependiendo del grado de polimerización y de sustitución, constituyendo soluciones más o menos viscosas; su solubilidad en agua es baja y es insoluble en etanol por lo que en el vino será, también, poco soluble dificultándose la dosificación del producto y las operaciones de limpieza. Para mejorar estos aspectos se han desarrollado productos líquidos eficaces [3].

Debido a su estructura molecular, la CMC, se une a la superficie del bitartrato potásico disuelto impidiendo el crecimiento de cristales por lo que actúa como un coloide protector; su ventaja es su bajo coste y que no es sensible a la temperatura. No obstante, también se han descrito algunos inconvenientes con respecto al uso de la CMC, como son, por ejemplo, que no puede ser utilizada en vinos tintos dado que precipita en presencia de taninos y para obviar este problema se ha propuesto la utilización conjunta de CMC con goma arábiga para la estabilización de vinos tintos, aunque los resultados no son siempre satisfactorios.

El poliaspartato de potasio (PA) es un polímero obtenido por condensación del ácido aspártico. El material original para la extracción del ácido aspártico es la pulpa de remolacha después de la extracción del azúcar, es por tanto, el subproducto de otra industria. Es completamente biodegradable. El efecto inhibidor del KA en la formación de cristales de tartrato, se basa en la adsorción de las moléculas del polímero en los sitios activos de crecimiento de la matriz del cristal. Esto conduce a una nucleación y crecimiento reducidos y a la formación de estructuras cristalinas distorsionadas [4].

En este estudio, se han tratado con CMC, PA y con RIC vinos blancos, rosados y dos tipos diferentes de vinos tintos y se evaluaron sus características tres meses después del tratamiento de estabilización. El objetivo es optimizar el tratamiento de estabilización tartárica de nuestros vinos con tratamientos alternativos al uso de las bajas temperaturas, para minimizar el coste del proceso y los efectos negativos sobre la calidad, pero manteniendo una adecuada estabilidad tartárica de los vinos. Se estudiaron las características enológicas y cromáticas de los vinos y se evaluaron también sensorialmente.

\section{Materiales y métodos}

El estudio se ha realizado con cuatro vinos diferentes elaborados en la Bodega Hacienda del Carche (Jumilla, Murcia, España), y que incluyen tres vinos de la vendimia del año 2017 (un blanco, un rosado y un tinto) y un tinto de la vendimia del año 2016.

Los tratamientos de estabilización se llevaron a cabo por la adición a cada vino, por un lado, de CMC líquida (Cellogum LV 20, Enartis) a $50 \mathrm{~mL} / \mathrm{HL}$ para los vinos blancos y rosados y una CMC con goma arábiga para los vinos tintos (Stab Mega, Enartis) a una dosis de $150 \mathrm{~mL} / \mathrm{HL}$; y, por otro lado, de poliaspartato potásico líquido a $100 \mathrm{~mL} / \mathrm{HL}$ para los vinos blancos y rosados (Zenith White, Enartis), y a una dosis de $200 \mathrm{~mL} / \mathrm{HL}$ para los vinos tintos (Zenith Color, Enartis). Para ambos casos, un vino al que no se le adicionó ningún producto se utilizó como control.

Para el tratamiento con resinas de intercambio iónico se trataron 10000 litros de vino de cada tipo. Se utilizó un sistema de intercambio catiónico semiautomático ISR (AEB Iberica). El ciclo de funcionamiento tiene una duración de 4 horas y se desarrolla en tres fases: acondicionamiento - regeneración - lavado. En este tipo de tratamientos se trata solo parte vino para mezclarlo con el vino original hasta alcanzar una estabilidad. Normalmente los \% de mezcla que se suelen barajar es de entre un 10-20\%. Se considera que un vino es estable cuando la caída de conductividad es inferior a $3 \%$ en el test de Boulton. En nuestro ensayo se determinó que las mezclas que se deben hacer son las siguientes:

$$
\begin{aligned}
& \text { - 40\% Vino Blanco Testigo }+60 \% \text { Resina. } \\
& -50 \% \text { Vino Rosado Testigo }+50 \% \text { Resina. } \\
& -70 \% \text { Vino Tinto } 2017 \text { Testigo }+30 \% \text { Resina. } \\
& -80 \% \text { Vino Tinto } 2016 \text { Testigo }+20 \% \text { Resina. }
\end{aligned}
$$

Tras los tratamientos, los vinos fueron embotellados y los análisis se realizaron por triplicado a los tres meses del embotellado.

\subsection{Determinaciones en los vinos}

Los parámetros enológicos generales ( $\mathrm{pH}$, acidez total, grado alcohólico) se determinaron de acuerdo a los métodos oficiales de la OIV.

La conductividad se determinó en un conductímetro Sension EC71. 
El contenido en minerales se determina por espectrometría de masas de plasma acoplado inductivamente (ICP-MS) (Agilent 7900, Tokio, Japón). Las muestras de vino se diluyen $(1: 10 \mathrm{v} / \mathrm{v})$ con $2 \% \mathrm{v} / \mathrm{v}$ de HNO3. Se utiliza una curva de calibración de hasta $100 \mathrm{mg} / \mathrm{L}$ para Na, $\mathrm{K}$ y Ca.

La estabilidad tartárica se determinó por el Test de Boulton. Es un ensayo analítico que consiste en una precipitación rápida de los cristales de bitartrato de potasio, que se hallan sobresaturados en el vino. La muestra del vino objeto del análisis se enfría a $0{ }^{\circ} \mathrm{C}$. y se provoca una precipitación rápida de los cristales por adición de $10 \mathrm{~g} / 1$ aproximados de un reactivo de bitartrato de potasio en polvo, siguiendo la disminución del potasio por un método conductométrico. Cuando la sobresaturación se reduce a cero, ya no hay más precipitación y el valor de la conductividad permanece constante. La muestra tendrá en estos momentos las características de un vino estable, y esta conductividad es la que debe tenerse en cuenta. Se utilizó un sistema criotermostático (JP Selecta, España).

Los ácidos tartárico y málico se determinaron utilizando sendos kits enzimáticos (Boehringer Mannheim R-Biopharm, Alemania). Todas las medidas espectrofotométricas se realizaron en un espectrofotómetro $\mathrm{HE} \lambda \mathrm{IOS} \alpha$ (TermoSpectronic, USA) a partir de las diferentes muestras filtradas usando filtros de nylon de 0.45 micras.

\subsubsection{Determinaciones cromáticas}

Índice de polifenoles totales (IPT): Se obtiene por medida de la absorbancia a $280 \mathrm{~nm}$ del vino diluido 100 veces con cubetas de $1 \mathrm{~cm}$ de paso óptico.

Antocianos totales: se obtiene tras la adición de $20 \mathrm{~mL}$ de HCL $0.1 \mathrm{~N}$ a $0.5 \mathrm{~mL}$ de vino. Pasados 30 minutos se mide la absorbancia a $520 \mathrm{~mm}$ en cubetas de $1 \mathrm{~cm}$ de paso óptico.

Antocianos poliméricos: se determinaron mediante la adición de $160 \mu \mathrm{L}$ de $\mathrm{SO}_{2}$ al $5 \%$ a $2 \mathrm{~mL}$ de muestra. La muestra fue agitada y tras 1 minuto se midió la absorbancia a $520 \mathrm{~nm}$ en cubetas de $0.2 \mathrm{~cm}$ de paso óptico.

Intensidad de color (IC): Se determina a través de la suma de las absorbancias a $620 \mathrm{~nm}$ (componente azul), $520 \mathrm{~nm}$ (componente roja) y $420 \mathrm{~nm}$ (componente amarilla) la intensidad de color del vino sin diluir mediante cubetas de $0.2 \mathrm{~cm}$ de paso óptico.

Taninos totales: La determinación de taninos totales se llevó a cabo por el método de la metilcelulosa [6]. Para ello, a $50 \mu \mathrm{L}$ de vino se le adicionan $600 \mu \mathrm{L}$ de una solución de metil-celulosa $(0.04 \%)$, se deja reposar 2-3 minutos y a continuación se le adicionan $400 \mu \mathrm{L}$ de una solución saturada de sulfato de amonio y $800 \mu \mathrm{L}$ de $\mathrm{H}_{2} \mathrm{O}$. La muestra se agita y se deja reposar 10 minutos. Después se centrifuga a $10000 \mathrm{rpm}$ durante 5 minutos y se mide la absorbancia a $280 \mathrm{~nm}$. Una muestra control sin metilcelulosa es requerida para determinar la absorbancia correspondiente a los taninos. La concentración de estos compuestos se expresa en $\mathrm{mg} / \mathrm{L}$ utilizando la (-)epicatequina como patrón externo.

\subsubsection{Análisis sensorial}

Se realizó una prueba triangular para determinar si existían diferencias entre las características de los vinos
Tabla 1. Vino blanco.

\begin{tabular}{|c|c|c|c|c|c|}
\hline & Testigo & CMC & PA & RIC \\
\hline \multicolumn{2}{|l|}{$\mathrm{pH}$} & $3.62 \mathrm{~b}$ & $3.55 \mathrm{~b}$ & $3.54 \mathrm{~b}$ & $3.02 \mathrm{a}$ \\
\hline \multicolumn{2}{|c|}{ Acidez Total (g/l) } & $4.56 \mathrm{a}$ & $5.38 \mathrm{~b}$ & $5.34 \mathrm{~b}$ & $6.83 \mathrm{c}$ \\
\hline \multicolumn{2}{|c|}{ Conductividad } & $1.91 \mathrm{~b}$ & $2.24 \mathrm{c}$ & $2.26 \mathrm{c}$ & $1.41 \mathrm{a}$ \\
\hline \multicolumn{2}{|l|}{ IC } & $0.51 \mathrm{~b}$ & $0.54 \mathrm{~b}$ & $0.52 \mathrm{~b}$ & $0.11 \mathrm{a}$ \\
\hline \multicolumn{2}{|l|}{ Tono } & $3.51 \mathrm{a}$ & $3.45 \mathrm{a}$ & $3.58 \mathrm{a}$ & $3.48 \mathrm{a}$ \\
\hline \multicolumn{2}{|c|}{ Ac. Málico (g/l) } & $0.0 \mathrm{a}$ & $0.1 \mathrm{c}$ & $0.1 \mathrm{bc}$ & $0.1 \mathrm{~b}$ \\
\hline \multicolumn{2}{|c|}{ Ac. Tartárico (g/l) } & $3.38 \mathrm{a}$ & $4.33 \mathrm{~b}$ & $4.59 \mathrm{~b}$ & $3.93 \mathrm{ab}$ \\
\hline \multirow{3}{*}{$\begin{array}{l}\text { Minerales } \\
(\mathrm{ppm})\end{array}$} & $\mathrm{Ca}$ & $85.93 \mathrm{~b}$ & $83.75 \mathrm{~b}$ & $90.66 \mathrm{~b}$ & $36.41 \mathrm{a}$ \\
\hline & $\mathrm{Na}$ & $21.15 \mathrm{a}$ & $25.39 \mathrm{~b}$ & $25.57 \mathrm{~b}$ & $41.24 \mathrm{c}$ \\
\hline & $\mathrm{K}$ & $853.1 \mathrm{~b}$ & $929.3 \mathrm{c}$ & $969.9 \mathrm{c}$ & $398.5 \mathrm{a}$ \\
\hline
\end{tabular}

CMC: carboximetilcelulosa, PA: poliaspartato de potasio, RIC: resina de intercambio catiónico.

IC: Intensidad de Color, IPT: Índice de Polifenoles Totales, AT: Antocianos Totales, AP: Antocianos Poliméricos, TT: Taninos Totales.

Las medias con letras distintas son diferentes significativamente a $\mathrm{p}$ valor $<0.05$

estabilizados con respecto a su correspondiente vino control. Es una prueba de diferenciación en la que se presentan al catador tres muestras codificadas, dos de las cuales son iguales, y se le pide que indique cual es la muestra diferente Los catadores utilizados en esta prueba fueron doce, con un entrenamiento previo básico.

\section{Resultados y discusión}

Los resultados de los diferentes vinos los encontramos en las Tablas 1, 2, 3 y 4. Se puede observar con el $\mathrm{pH}$ baja en todos los vinos tras los tratamientos, sobre todo en los vinos estabilizados por resinas de intercambio catiónico y sobre todo en blancos y rosados. El vino tratado con RIC se mezcló con el vino control hasta conseguir un valor en el test de Boulton que asegure la estabilidad del vino, aunque para ello en casi todos los vinos el descenso del $\mathrm{pH}$ es superior al admitido. El valor de la conductividad en los vinos sube ligeramente para los vinos tratados con CMC y PA y baja en los vinos tratados con RIC, por la eliminación de Ca y K observada. Se han observado pocos efectos sobre el ácido tartárico y el málico. Sin embargo [3] encontró una disminución de conductividad cuando utilizó CMC para estabilizar diversos vinos. [5] también reportó que la eliminación de potasio con el uso de RIC ayuda a la estabilización del vino y no es único catión que se ve afectado ya que otros cationes como el calcio, hierro y manganeso también se pueden ver afectados.

Las características cromáticas de los vinos tintos se han visto poco afectadas por los distintos tratamientos. En el vino tinto de la vendimia de 2017 no se ha observado perdida de IC ni del contenido en polifenoles totales tras los tratamientos. [4] también encontró que PA no afecta a la composición cromática de los vinos tratados. Si se ha observado una caída de la concentración de antocianos con las RIC y lo más significativo ha sido la caída de taninos con las RIC. Los vinos elaborados en la vendimia de 2016 se han visto mucho menos afectados por los tratamientos, no observándose disminuciones ni en el color, ni antocianos ni taninos.

[2] también encontraron que el uso de RIC para la estabilización de vinos tintos causó un descenso significativo de antocianos y de las diferentes fracciones de proantocianidinas. 
Tabla 2. Vino rosado.

\begin{tabular}{|c|c|c|c|c|c|}
\hline & Testigo & CMC & $\mathbf{P A}$ & RIC \\
\hline \multicolumn{2}{|l|}{$\mathrm{pH}$} & $3.49 \mathrm{~b}$ & $3.53 \mathrm{c}$ & $3.53 \mathrm{c}$ & $3.09 \mathrm{a}$ \\
\hline \multicolumn{2}{|c|}{ Acidez Total $(\mathrm{g} / \mathrm{l})$} & $3.90 \mathrm{a}$ & $4.11 \mathrm{a}$ & $4.16 \mathrm{a}$ & $5.15 \mathrm{~b}$ \\
\hline \multicolumn{2}{|c|}{ Conductividad } & $1.33 \mathrm{~b}$ & $1.71 \mathrm{c}$ & $1.73 \mathrm{c}$ & $1.24 \mathrm{a}$ \\
\hline \multicolumn{2}{|l|}{ IC } & $2.16 \mathrm{~b}$ & $2.23 \mathrm{c}$ & $2.30 \mathrm{~d}$ & $0.57 \mathrm{a}$ \\
\hline \multicolumn{2}{|l|}{ Tono } & $0.82 \mathrm{~b}$ & $0.82 \mathrm{~b}$ & $0.83 \mathrm{~b}$ & $0.65 \mathrm{a}$ \\
\hline \multicolumn{2}{|l|}{ IPT } & $9.34 \mathrm{~b}$ & $9.21 \mathrm{ab}$ & $9.81 \mathrm{~b}$ & $8.67 \mathrm{a}$ \\
\hline \multicolumn{2}{|l|}{ AT (mg/l) } & $31.94 \mathrm{~b}$ & $30.80 \mathrm{~b}$ & $31.67 \mathrm{~b}$ & $23.44 \mathrm{a}$ \\
\hline \multicolumn{2}{|l|}{$\mathrm{AP}(\mathrm{mg} / \mathrm{l})$} & $1.48 \mathrm{~b}$ & $1.34 \mathrm{~b}$ & $2.02 \mathrm{c}$ & $1.13 \mathrm{a}$ \\
\hline \multicolumn{2}{|c|}{ Ac. Málico $(\mathrm{g} / \mathrm{l})$} & $0.03 \mathrm{a}$ & $0.04 \mathrm{a}$ & $0.02 \mathrm{a}$ & $0.02 \mathrm{a}$ \\
\hline \multicolumn{2}{|c|}{ Ac. Tartárico(g/l) } & $3.92 \mathrm{a}$ & $4.22 \mathrm{a}$ & $4.66 \mathrm{a}$ & $4.46 \mathrm{a}$ \\
\hline \multirow{3}{*}{$\begin{array}{c}\text { Minerales } \\
(\mathrm{ppm})\end{array}$} & $\mathrm{Ca}$ & $48.37 \mathrm{~b}$ & $51.53 \mathrm{~b}$ & $51.40 \mathrm{~b}$ & $30.37 \mathrm{a}$ \\
\hline & $\mathrm{Na}$ & $22.85 \mathrm{a}$ & $32.06 \mathrm{bc}$ & $29.27 \mathrm{~b}$ & $35.82 \mathrm{c}$ \\
\hline & $\mathrm{K}$ & $700.5 \mathrm{~b}$ & $714.4 \mathrm{~b}$ & $721.8 \mathrm{~b}$ & $432.8 \mathrm{a}$ \\
\hline
\end{tabular}

CMC: carboximetilcelulosa, PA: poliaspartato de potasio, RIC: resina de intercambio catiónico.

IC: Intensidad de Color, IPT: Índice de Polifenoles Totales, AT: Antocianos Totales, AP: Antocianos Poliméricos, TT: Taninos Totales

Las medias con letras distintas son diferentes significativamente a $p$ valor $<0.05$.

Tabla 3. Vino tinto 2017.

\begin{tabular}{|c|c|c|c|c|c|}
\hline & Testigo & CMC & $\mathbf{P A}$ & RIC \\
\hline \multicolumn{2}{|l|}{$\mathrm{pH}$} & $3.82 \mathrm{~b}$ & $3.82 \mathrm{~b}$ & $3.81 \mathrm{~b}$ & $3.57 \mathrm{a}$ \\
\hline \multicolumn{2}{|c|}{ Acidez Total $(\mathrm{g} / \mathrm{l})$} & $5.09 \mathrm{a}$ & $5.25 \mathrm{a}$ & $5.21 \mathrm{a}$ & $5.99 \mathrm{~b}$ \\
\hline \multicolumn{2}{|c|}{ Conductividad } & $2.52 \mathrm{~b}$ & $2.6 \mathrm{c}$ & $2.63 \mathrm{c}$ & $2.03 \mathrm{a}$ \\
\hline \multicolumn{2}{|l|}{ IC } & $13.07 \mathrm{a}$ & $14.27 \mathrm{ab}$ & $14.95 \mathrm{~b}$ & 13.90ab \\
\hline \multicolumn{2}{|l|}{ Tono } & $0.70 \mathrm{bc}$ & $0.70 \mathrm{~b}$ & $0.72 \mathrm{c}$ & $0.67 \mathrm{a}$ \\
\hline \multicolumn{2}{|l|}{ IPT } & $69.81 \mathrm{a}$ & $74.98 \mathrm{~b}$ & $74.56 \mathrm{~b}$ & $69.89 \mathrm{a}$ \\
\hline \multicolumn{2}{|l|}{ AT (mg/l) } & $399.13 \mathrm{~b}$ & $431.49 \mathrm{c}$ & $435.7 \mathrm{c}$ & $325.5 \mathrm{a}$ \\
\hline \multicolumn{2}{|l|}{$\mathrm{AP}(\mathrm{mg} / \mathrm{l})$} & $70.17 \mathrm{~b}$ & $72.67 \mathrm{bc}$ & $77.5 \mathrm{c}$ & $60.03 \mathrm{a}$ \\
\hline \multicolumn{2}{|l|}{ TT (mg/l) } & $1820.3 \mathrm{~b}$ & $1749.5 \mathrm{~b}$ & $1835.2 b$ & $1195.5 \mathrm{a}$ \\
\hline \multicolumn{2}{|c|}{ Ac. Málico (g/l) } & $0.09 \mathrm{a}$ & $0.10 \mathrm{a}$ & $0.07 \mathrm{a}$ & $0.09 \mathrm{a}$ \\
\hline \multicolumn{2}{|c|}{ Ac. Tartárico(g/l) } & $4.01 \mathrm{a}$ & $4.30 \mathrm{a}$ & $4.99 \mathrm{a}$ & $4.30 \mathrm{a}$ \\
\hline \multirow{3}{*}{$\begin{array}{c}\text { Minerales } \\
(\mathrm{ppm})\end{array}$} & $\mathrm{Ca}$ & $68.97 \mathrm{~b}$ & $70.84 \mathrm{~b}$ & $72.53 \mathrm{~b}$ & $53.35 \mathrm{a}$ \\
\hline & $\mathrm{Na}$ & $16.33 \mathrm{a}$ & $24.19 \mathrm{c}$ & $19.88 \mathrm{~b}$ & $24.07 \mathrm{c}$ \\
\hline & $\mathrm{K}$ & $1146.0 \mathrm{ab}$ & $1262.6 \mathrm{~b} \mathrm{c}$ & $1323.7 \mathrm{c}$ & $1034.1 \mathrm{a}$ \\
\hline
\end{tabular}

CMC: carboximetilcelulosa, PA: poliaspartato de potasio, RIC: resina de intercambio catiónico.

IC: Intensidad de Color, IPT: Índice de Polifenoles Totales, AT: Antocianos Totales, AP: Antocianos Poliméricos, TT: Taninos Totales Las medias con letras distintas son diferentes significativamente a $p$ valor $<0.05$.

Las Figs. 1, 2, 3 y 4 muestran los valores obtenidos con el Test de Boulton justo tras los tratamientos y a los tres meses del embotellado. Los vinos blancos y rosados control son los más inestables mientras que casi todos los vinos tratados alcanzan valores por debajo del $3 \%$ lo que los convierte en muy estables. Solo el vino rosado tratado con PA y el tinto 2017 con RIC muestran valores algo superiores al $3 \%$ pero inferiores al 5\%. Los resultados de [aspraudi] también mostraron la excelente efectividad de PA para limitar la precipitación tartárica en vinos de diferente composición físico-química.

[6] también ensayó PA y comparó los resultados con los vinos tratados con ácido metatartárico. A los 12 meses, tanto en el caso del vino blanco como en el del vino tinto, el MTA había perdido su eficacia como estabilizante, pero, por el contrario, los vinos tratados con PA eran todavía estables tartáricamente. Resultados similares los encontró [4].
Tabla 4. Vino tinto 2016.

\begin{tabular}{|c|c|c|c|c|c|}
\hline & Testigo & CMC & $\mathbf{P A}$ & RIC \\
\hline \multicolumn{2}{|l|}{$\mathrm{pH}$} & $3.79 \mathrm{~b}$ & $3.60 \mathrm{ab}$ & $3.78 \mathrm{~b}$ & $3.39 \mathrm{a}$ \\
\hline \multicolumn{2}{|c|}{ Acidez Total $(\mathrm{g} / \mathrm{l})$} & $4.77 \mathrm{a}$ & $4.99 \mathrm{~b}$ & $4.91 \mathrm{~b}$ & $5.58 \mathrm{c}$ \\
\hline \multicolumn{2}{|c|}{ Conductividad } & $2.42 \mathrm{c}$ & $2.29 \mathrm{~b}$ & $2.61 \mathrm{~d}$ & $1.94 \mathrm{a}$ \\
\hline \multicolumn{2}{|l|}{ IC } & $9.55 \mathrm{a}$ & $10.36 \mathrm{~b}$ & $10.38 \mathrm{~b}$ & $10.53 \mathrm{~b}$ \\
\hline \multicolumn{2}{|l|}{ Tono } & $0.82 \mathrm{c}$ & $0.77 \mathrm{~b}$ & $0.81 \mathrm{c}$ & $0.72 \mathrm{a}$ \\
\hline \multicolumn{2}{|l|}{ IPT } & $52.44 \mathrm{a}$ & $55.80 \mathrm{~b}$ & $51.92 \mathrm{a}$ & $55.14 \mathrm{~b}$ \\
\hline \multicolumn{2}{|l|}{$\mathrm{AT}(\mathrm{mg} / \mathrm{l})$} & $216.0 \mathrm{a}$ & $234.8 \mathrm{~b}$ & $249.0 \mathrm{c}$ & $224.5 \mathrm{ab}$ \\
\hline \multicolumn{2}{|l|}{$\mathrm{AP}(\mathrm{mg} / \mathrm{l})$} & $43.36 \mathrm{a}$ & $47.30 \mathrm{c}$ & $45.89 \mathrm{~b}$ & $47.46 \mathrm{c}$ \\
\hline \multicolumn{2}{|l|}{ TT (mg/l) } & $1464.1 \mathrm{a}$ & $1277.2 \mathrm{a}$ & $1232.4 \mathrm{a}$ & $1594.7 \mathrm{a}$ \\
\hline \multicolumn{2}{|c|}{ Ácido Málico } & $0.03 \mathrm{a}$ & $0.04 \mathrm{a}$ & $0.01 \mathrm{a}$ & $0.05 \mathrm{a}$ \\
\hline \multicolumn{2}{|c|}{ Ácido Tartárico } & $3.99 \mathrm{a}$ & $3.87 \mathrm{a}$ & $4.41 \mathrm{a}$ & $4.15 \mathrm{a}$ \\
\hline \multirow{3}{*}{$\begin{array}{l}\text { Minerales } \\
\quad(\mathrm{ppm})\end{array}$} & $\mathrm{Ca}$ & $58.20 \mathrm{ab}$ & $59.14 \mathrm{ab}$ & $69.70 \mathrm{~b}$ & $45.73 \mathrm{a}$ \\
\hline & $\mathrm{Na}$ & $12.63 \mathrm{a}$ & $16.56 \mathrm{ab}$ & $19.08 \mathrm{~b}$ & $17.70 \mathrm{ab}$ \\
\hline & $\mathrm{K}$ & $1143.5 \mathrm{~b}$ & $1053.0 \mathrm{~b}$ & $1455.0 \mathrm{~b}$ & $853.4 \mathrm{a}$ \\
\hline
\end{tabular}

CMC: carboximetilcelulosa, PA: poliaspartato de potasio, RIC: resina de intercambio catiónico.

IC: Intensidad de Color, IPT: Índice de Polifenoles Totales, AT: Antocianos Totales, AP: Antocianos Poliméricos, TT: Taninos Totales.

Las medias con letras distintas son diferentes significativamente a $p$ valor $<0.05$.

\section{Vino blanco}

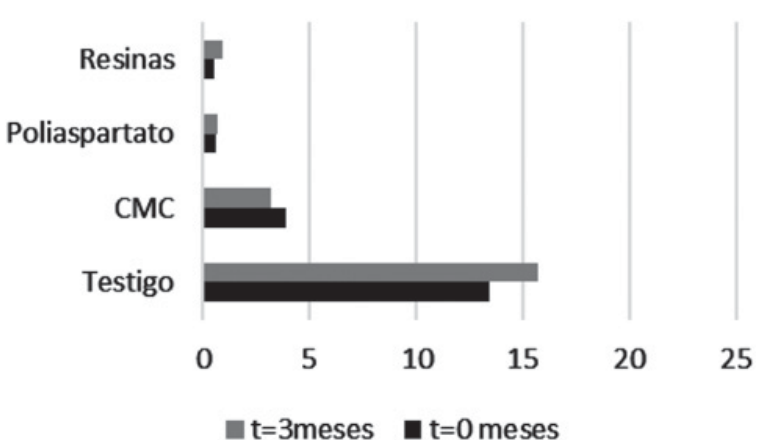

Figura 1. Valores obtenidos al realizar el test de Boulton en vinos blancos a tiempo 0 y a los tres meses del embotellado.

\section{Vino rosado}

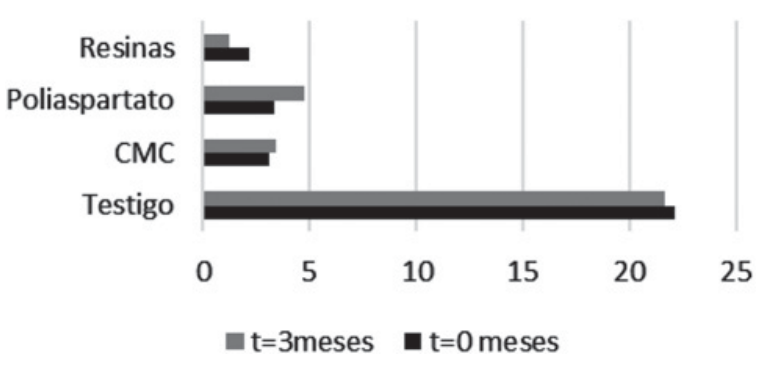

Figura 2. Valores obtenidos al realizar el test de Boulton en vinos rosados a tiempo 0 y a los tres meses del embotellado.

La Tabla 5 muestra los resultados de la cata triangular efectuada con los vinos, enfrentando todos los vinos tratados con su correspondiente control. En los vinos blancos los catadores fueron capaces de diferenciar todos los vinos tratados de sus respectivos controles, siendo preferido el vino testigo al estabilizado por CMC y RIC y el vino estabilizado por PA fue el preferido. Cuando se evaluó el vino rosado, los catadores no pudieron 


\section{Vino tinto 2017}

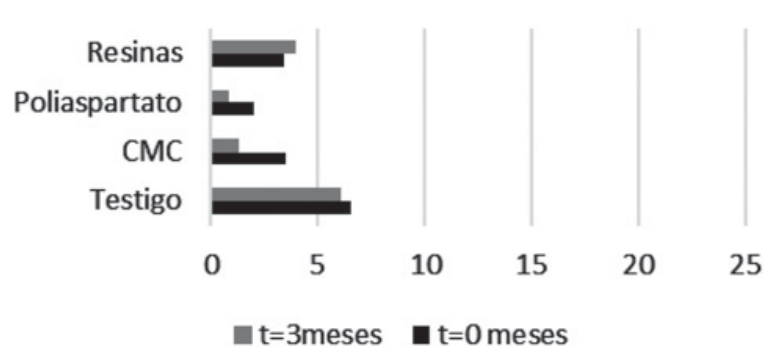

Figura 3. Valores obtenidos al realizar el test de Boulton en vinos tintos de la vendimia 2017 a tiempo 0 y a los tres meses del embotellado.

\section{Vino tinto 2016}

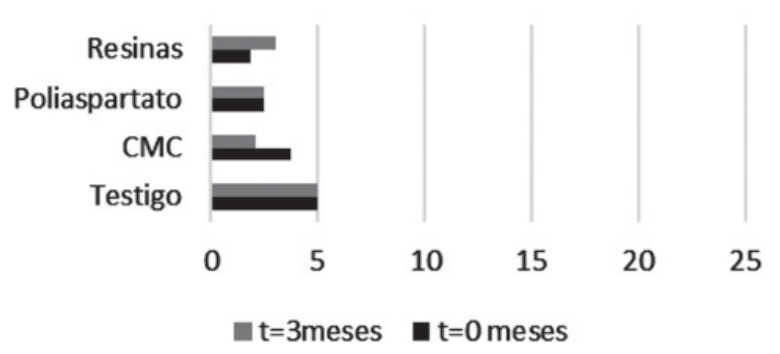

Figura 4. Valores obtenidos al realizar el test de Boulton en vinos tintos de la vendimia 2016 a tiempo 0 y a los tres meses del embotellado.

diferenciar entre el vino testigo y el tratado con PA, prefirieron el vino tratado con CMC frente al testigo $\mathrm{y}$ el testigo frente al tratado por RIC. En ambos vinos tintos, los catadores no pudieron distinguir entre los vinos tratados por CMC y su testigo, si diferenciaron los vinos tratados por PA, siendo estos los preferidos, y los vinos tratados por RIC, donde el control fue el preferido.

\section{Conclusiones}

Todos los tratamientos fueron efectivos para estabilizar el vino a tiempo $0 \mathrm{y}$ a tres meses, originando pocos cambios en los parámetros cromáticos de los vinos. Los vinos tratados con PA consiguieron una alta estabilización y en general fueron los preferidos por los catadores. Los vinos que mostraron los peores resultados cromáticos y sensoriales fueron los vinos tratados por RIC. Por tanto, la CMC y sobretodo el PA resultan muy adecuados para
Tabla 5. Resultados de la cata triangular efectuada con 10 catadores.

\begin{tabular}{|c|c|c|}
\hline & Número de aciertos & Preferencias \\
\hline \multicolumn{3}{|l|}{ Vino Blanco } \\
\hline Testigo vs CMC & 6 & \\
\hline Testigo vs PA & 8* & PA (6) \\
\hline Testigo vs RIC & $10 * *$ & Testigo (10) \\
\hline \multicolumn{3}{|l|}{ Vino Rosado } \\
\hline Testigo vs CMC & $8 *$ & CMC (5) \\
\hline Testigo vs PA & 6 & \\
\hline Testigo vs RIC & $10 * *$ & Testigo (10) \\
\hline \multicolumn{3}{|l|}{ Vino tinto 2016} \\
\hline Testigo vs CMC & 0 & \\
\hline Testigo vs PA & $10 * *$ & PA (8) \\
\hline Testigo vs RIC & 8* & Testigo (8) \\
\hline \multicolumn{3}{|l|}{ Vino tinto 2017} \\
\hline Testigo vs CMC & 4 & \\
\hline Testigo vs PA & $10 * *$ & PA (8) \\
\hline Testigo vs RIC & $10 * *$ & RIC (6) \\
\hline
\end{tabular}

estabilización de todo tipo de vinos. Es necesario observar durante más tiempo la evolución de estos vinos para realmente poder evaluar todo su potencial.

\section{Referencias}

[1] C. Lasanta, J. Gomez, Trends Food Sci. Technol. 28 (2012)

[2] V. Ibeas, A.C. Correia, A. Jordão, Food Res. Int. 69, 364 (2015)

[3] R. Guise, L. Filipe-Ribeiro, D. Nascimento, O. Bessa, F.M. Nunes, F. Cosme, Food Chem. 156, 250 (2014)

[4] A. Bosso, L. Panero, M. Petrozziello, M. Sollazzo, A. Asproudi, S. Motta, Food Chem. 185, 1 (2015)

[5] M.J. Cabrita, R. Garcia, S. Catarino, Recent Advances in Wine Stabilization (Nova Science Publishers, Inc., 2016)

[6] R. López, E. Vela, P. Hernández-Orte, V. Ferreira, En: Enologia 2015 (Innovación vitivinícola, Ed. Univ. Rovira y Virgilli, 2015) 